\title{
Search for Orbital Motion of the Pulsar 4U 1626-67: Candidate for a Neutron Star with a Supernova Fall-back Accretion Disk
}

\author{
Chetana Jain ${ }^{1, *}$, Biswajit Paul ${ }^{2}$, Kaustubh Joshi $^{3}$, Anjan Dutta ${ }^{1} \&$ \\ Harsha Raichur ${ }^{2,4}$ \\ ${ }^{1}$ Department of Physics and Astrophysics, University of Delhi, Delhi 110 007, India. \\ ${ }^{2}$ Raman Research Institute, Sadashivnagar, C. V. Raman Avenue, Bangalore 560 080, India. \\ ${ }^{3}$ Department of Electrical Engineering, Indian Institute of Technology - Bombay, Powai, \\ Mumbai 400 076, India. \\ ${ }^{4}$ Joint Astronomy Programme, Indian Institute of Science, Bangalore 560 012, India. \\ *e-mail: chetanajain11@yahoo.co.in
}

Received 2007 June 1; revised 2008 January 31; accepted 2008 February 1

\begin{abstract}
We report here results from a new search for orbital motion of the accretion powered X-ray pulsar 4U 1626-67 using two different analysis techniques. X-ray light curve obtained with the Proportional Counter Array of the Rossi X-ray Timing Explorer during a long observation carried out in February 1996, was used in this work. The spin period and the local period derivative were first determined from the broad $2-60 \mathrm{keV}$ energy band light curve and these were used for all subsequent timing analysis. In the first technique, the orbital phase dependent pulse arrival times were determined for different trial orbital periods in the range of 500 to $10,000 \mathrm{~s}$. We have determined a $3 \sigma$ upper limit of $13 \mathrm{lt}-\mathrm{ms}$ on the projected semimajor axis of the orbit of the neutron star for most of the orbital period range, while in some narrow orbital period ranges, covering about $10 \%$ of the total orbital period range, it is $20 \mathrm{lt}-\mathrm{ms}$. In the second method, we have measured the pulse arrival times at intervals of $100 \mathrm{~s}$ over the entire duration of the observation. The pulse arrival time data were used to put an upper limit on any periodic arrival time delay using the Lomb-Scargle periodogram. We have obtained a similar upper limit of $10 \mathrm{lt}-\mathrm{ms}$ using the second method over the orbital period range of 500-10,000 s. This puts very stringent upper limits for the mass of the compact object except for the unlikely case of a complete face-on orientation of the binary system with respect to our line-of-sight. In the light of this measurement and the earlier reports, we discuss the possibility of this system being a neutron star with a supernovae fall-back accretion disk.
\end{abstract}

Key words. Accretion, accretion disks—binaries: general—stars: individual (4U 1626-67)—-stars: neutron-X-rays: stars. 


\section{Introduction}

$4 \mathrm{U} 1626-67$ is a very well studied accretion powered X-ray pulsar. The X-ray pulsations were discovered by Rappaport et al. (1977) during SAS3 observations. The presence of an accretion disk around the high magnetic field neutron star in this system is very well established from X-ray spectroscopic measurements and also from the detection of quasi-periodic oscillations in X-rays (Shinoda et al. 1990; Kaur et al. 2008). The X-ray spectrum of $4 \mathrm{U} 1626-67$ was studied in detail first with the ASCA spectrometers (Angelini et al. 1995) and later with the High Energy Transmission Grating instrument of Chandra (Schulz et al. 2001; Krauss et al. 2007). The spectrum shows clear signatures of irradiation of the accretion disk by the central X-ray source. The spectrum is rich in emission lines and also shows Doppler and thermal broadening of the line features. The neutron star has a magnetic field strength of a few times $10^{12}$ Gauss, determined from the cyclotron resonance absorption features in the hard X-ray band (Orlandini et al. 1998). In addition to the spectral signatures, the optical emission also shows reprocessing of the X-ray pulses. The Fourier power spectrum of the optical light curve of $4 \mathrm{U}$ 1626-67 clearly shows the reprocessed X-ray pulses at $130.38 \mathrm{mHz}$ (Middleditch et al. 1981; Chakrabarty et al. 2001). Additionally, the pulse peak in the power spectrum has multiple sidebands, the main ones are separated by about $0.4 \mathrm{mHz}$. If the sidebands in the power spectrum are taken to be signatures of the binary companion, this indicates an orbital period of about $2485 \pm 60 \mathrm{~s}$. Based on these studies, it is generally felt that the neutron star most probably, has a low mass binary companion and a small orbital period of about 42 minutes. However, orbital motion of the neutron star (usually observed in binary pulsars in the form of Doppler shift in the pulse period, or delay in the pulse arrival time) has never been detected in $4 \mathrm{U}$ 1626-67. Using a long X-ray observation with the Medium Energy (ME) detectors of the EXOSAT observatory, Levine et al. (1988) determined an upper limit of 10 lt-ms for $a_{x} \sin (i)$, which is the most stringent upper limit reported so far for this object. In the present work, we have used the Lomb-Scargle periodogram technique for detection of a periodic signal in an unevenly sampled time series with RXTE-PCA. Compared to the Fourier transform, Lomb-Scargle method allows us to search for a periodic signal with the desired resolution in orbital period.

We have analyzed data of this pulsar from a very long observation carried out in February 1996, with the RXTE-PCA. We have used two different pulse arrival time analysis techniques to search for any possible binary motion of the neutron star. Results obtained from this long RXTE-PCA observation are presented here.

\section{Observations and analysis}

The source 4U 1626-67 was observed with RXTE from February 10, 1996 to February 14,1996 in three segments with useful exposures of $95561 \mathrm{~s}, 63393 \mathrm{~s}$ and $50255 \mathrm{~s}$ respectively. We have used light curves obtained with the Proportional Counter Array (PCA) of the RXTE, which has a total photon collection area of $6500 \mathrm{~cm}^{2}$ (Jahoda et al. 1996). This dataset is well suited for the search of orbital motion using the pulse arrival time measurements around the proposed orbital period of about 42 minutes. Light curves were extracted from data stored in the event mode, which has the highest energy and timing information. For data screening, the filtering criteria applied were: all the five Proportional Counter Units (PCU) were ON, time since South Atlantic Anomaly 

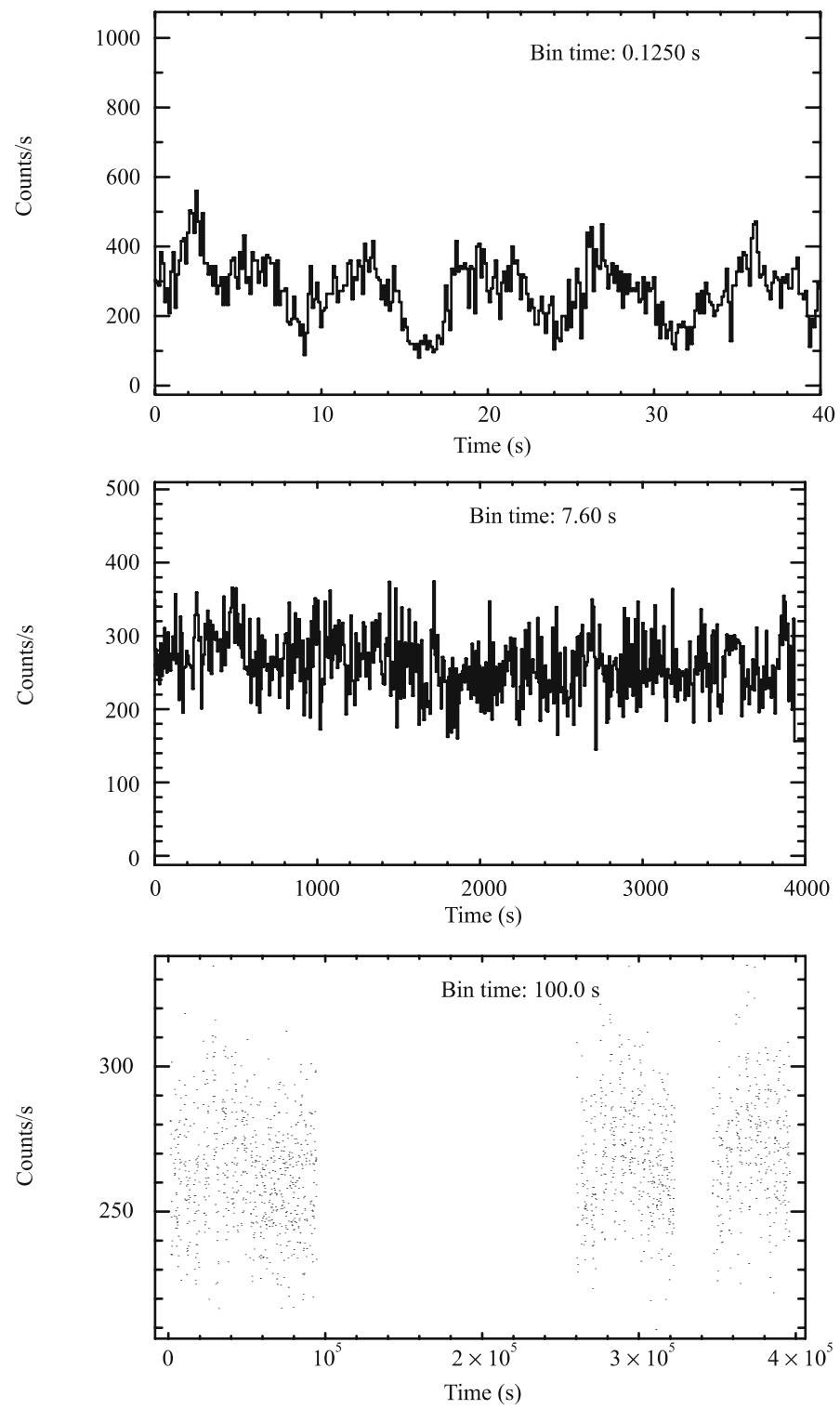

Figure 1. 2-60 keV RXTE/PCA light curves with different bin sizes for $4 \mathrm{U}$ 1626-67. The bottom panel shows the light curve obtained from the entire light curve. The upper and middle panels show a section of the light curve.

(SAA) was greater than 10 minutes, pointing offset was less than 0.02 degrees, and Earth elevation angle was greater than 10 degrees.

The $2-60 \mathrm{keV}$ light curve obtained from the entire observation is shown in the bottom panel of Fig. 1 with a time resolution of $100 \mathrm{~s}$. In the two other panels of the same figure, sections of the light curve are shown with a time resolution of $0.125 \mathrm{~s}$ and $7.6 \mathrm{~s}$ respectively. For all analysis described here, the photon arrival times were first converted to the solar system barycenter. The pulsar $4 \mathrm{U}$ 1626-67 shows monotonic 


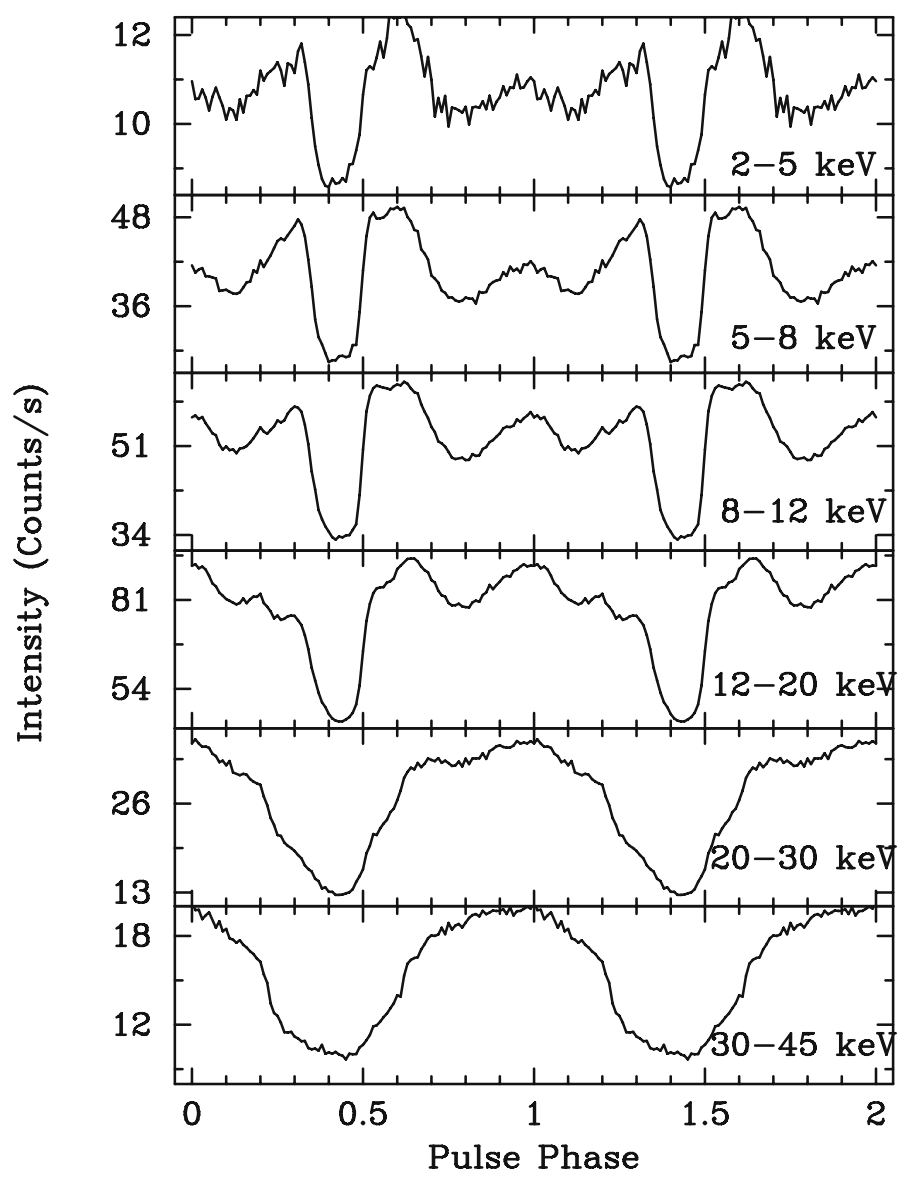

Figure 2. Energy-dependent pulse profiles of 4U 1626-67 covering an energy range of 2-45 keV. The highest signal-to-noise ratio is observed for $5-45 \mathrm{keV}$ energy band.

spin-up or spin-down for long periods (Chakrabarty et al. 1997). To establish the pulse period and the period derivative during the present observation, we have carried out the pulse folding and $\chi^{2}$ maximization analysis assuming different trial period derivatives (Paul et al. 2001). From this analysis we have determined a spin period of $7.66735 \mathrm{~s}(\mathrm{MJD}=50123.00)$ and a period derivative of $3.83 \times 10^{-11} \mathrm{~s} \mathrm{~s}^{-1}$. The period derivative derived here was used in all further analyses described below. These values are consistent with the same reported in Chakrabarty et al. (1997), derived as a long term solution of pulse period evolution of $4 \mathrm{U}$ 1626-67. The pulse profiles in different energy bands are shown in Fig. 2. The pulse profile of 4U 1626-67 is known to be energy dependent (Levine et al. 1988); bi-horned profile in 2-15 keV range and sinusoidal at higher energies. As shown in Fig. 2, at energies below $15 \mathrm{keV}$, we also found a sharp dip in the pulse profile in addition to bi-horned nature. From the Chandra-HETGS and XMM-RGS observations, Krauss et al. (2007), have also reported a dip in the pulse profile, after the 1990 torque reversal. The light curve has poor statistics below $5 \mathrm{keV}$ and there is a phase shift in the pulse profile above $10 \mathrm{keV}$. This source has a very hard spectrum, therefore, although the quantum efficiency of the PCA detectors drops 
rapidly after about $25 \mathrm{keV}$, we have observed the highest signal-to-noise ratio in the pulse profile for $5-45 \mathrm{keV}$ energy band. After examining the $\chi^{2}$ obtained from fitting a constant to the pulse profiles of various energy bands, we chose this wide energy band for pulse arrival time analysis.

\subsection{Pulse arrival time analysis with trial orbital periods}

To determine the orbital parameters of the system $4 \mathrm{U}$ 1626-67, we first searched for pulse arrival time delays as a function of the orbital period. To reduce the error of individual pulse arrival time measurements, we have added the pulse profiles coherently from identical orbital phases with trial orbital periods in the range of 500-10,000 s. The consecutive trial periods were chosen in a way to ensure that when data from this long RXTE observation are folded, the orbital phase error at the beginning and at the end is not more than 5\%. The entire observation duration was divided into segments of length equal to a trial orbital period. Each segment was further broken into 30 equal orbital phases. The ensemble of the corresponding phases from each orbital segment was then folded with the spin period and period derivative mentioned above and pulse profiles were created with 512 phase bins. A template profile was also created using the entire dataset. The advantage of using this template profile instead of any one of the phase specific profiles is better statistical accuracy. Finally we cross-correlated these orbital phase resolved pulse profiles with the template profile to determine the pulse arrival time delay. This process was repeated for the large number of trial orbital periods from $500-10,000 \mathrm{~s}$ in a fashion to avoid more than $5 \%$ error in orbital phase.

If any of the trial orbital period is close to the actual orbital period of the system, the plot of arrival times against the phase will show the pulse arrival time delay for a general elliptical orbit. The amplitude of the pulse arrival time delay should give us the projection of semi-major axis onto the line of sight $a_{x} \sin (i)$. We have also determined the orbital phase resolved pulse arrival times for 10 arbitrary trial orbital periods spread over the entire period range covered in the analysis. The average standard deviation of the 30 pulse arrival times for these arbitrary trial periods is found to be $0.002 \mathrm{~s}$, which was used as an error in the pulse arrival time measurement for all the other trial orbital periods. Subsequently, the relative pulse arrival times determined at 30 different orbital phases were fitted to a constant value. The $\chi^{2}$ derived from the constant fit for different trial orbital periods is shown in Fig. 3. It was observed that for most of the cases, the $\chi^{2}$ was less than 40 . But for some trial orbital periods, the $\chi^{2}$ was in the range of 40-70 for 29 degrees of freedom. We examined the pulse arrival time curve for these periods and also energy resolved data. From the nature of the pulse arrival times as a function of the trial orbital phase, it is evident that the high $\chi^{2}$ is due to random variation of pulse arrival times. The $1 \sigma$ and $3 \sigma$ upper limits of $a_{x} \sin (i)$, determined from the pulse arrival time analysis from this RXTE-PCA light curve of 4U 1626-67 are shown in Fig. 4 for a trial orbital period range of 500-10,000 s. As can be seen from the figure, we determined a $3 \sigma$ upper limit of $13 \mathrm{lt}-\mathrm{ms}$ on the projected semi-major axis of the orbit of the neutron star for most of the orbital period range, while in some narrow orbital period ranges, covering about $10 \%$ of the total orbital period range, it is $20 \mathrm{lt}-\mathrm{ms}$.

\subsection{Pulse arrival time analysis}

In this method, we searched for the presence of an orbital motion using the periodogram technique. We created continuous pulse profiles for about $100 \mathrm{~s}$ (13 pulses) in the 


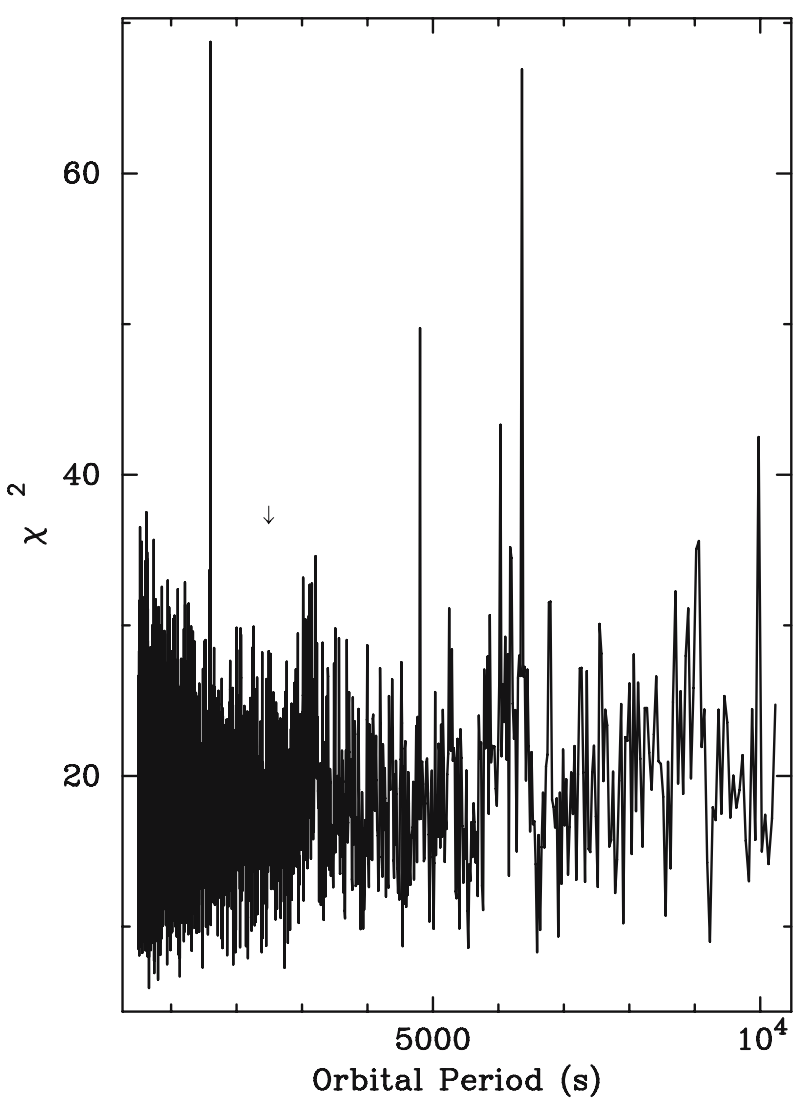

Figure 3. The $\chi^{2}$ values obtained after fitting a constant to the pulse arrival times at 30 different phases of the orbital period is shown here for trial orbital periods in the range of 500-10,000 s. The arrow marks the orbital period (2485 s) as inferred from the optical observations.

energy range of 5-45 keV. These pulse profiles were then cross-correlated with a master template pulse profile created using the entire stretch of observation. The peaks of the cross-correlation curves were used to determine the pulse arrival times in each interval of $100 \mathrm{~s}$ with respect to the phase coherent solution described by a spin period of $7.66735 \mathrm{~s}$ and a period derivative of $3.83 \times 10^{-11} \mathrm{~s} \mathrm{~s}^{-1}$. We searched for a periodic delay in the pulse arrival times using the Lomb-Scargle technique. The normalized Lomb-Scargle periodogram is shown in Fig. 5. There is an absence of a peak at any frequency and hence it rules out the possibility of any orbital motion. The frequency resolution chosen for the analysis was such that the total orbital phase offset is less than $1 \%$ of the trial orbital period, which is much less as compared to the earlier work. Figure 6 is a histogram of the power distribution made from the normalized periodogram. It follows an exponential distribution and a power of 13 or above in the simulated dataset would therefore indicate a signal detection. To set an upper limit on the projected semi-major axis of the orbit of the neutron star, we added sinusoidal signals of different amplitudes for periods of $500 \mathrm{~s}, 1250 \mathrm{~s}, 2500 \mathrm{~s}, 5000 \mathrm{~s}$ and 10,000 s to the entire data. As shown in Fig. 5, peaks were observed at the signal frequency for a signal amplitude of $10 \mathrm{~ms}$. There was no peak for an added sinusoidal signal of 


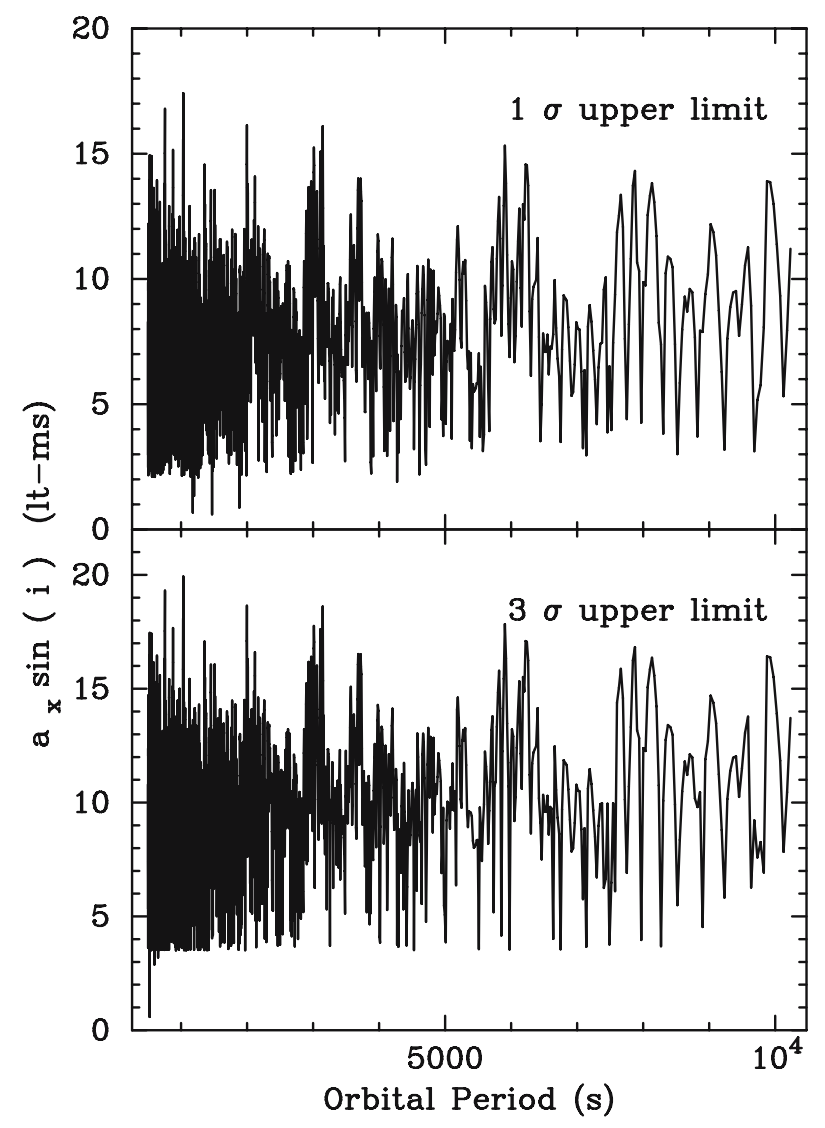

Figure 4. Upper limit of $a_{x} \sin (i)$ (in lt-ms) for different trial orbital periods. The top panel shows the $1 \sigma$ upper limit of $a_{x} \sin (i)$ while the bottom panel shows the $3 \sigma$ upper limits of $a_{x} \sin (i)$ for an orbital period range of 500-10,000 s.

amplitude less than $10 \mathrm{~ms}$. This sets an upper limit of $10 \mathrm{lt}-\mathrm{ms}$ on the projected semimajor axis of the orbit of the neutron star.

\section{Discussions}

The new upper limit of $10 \mathrm{lt}-\mathrm{ms}$ for $a_{x} \sin (i)$ of the persistent X-ray pulsar 4U 1626-67 gives a very small upper limit of $2.85 \times 10^{-6} \mathrm{M}_{\odot}$ for the mass function. This puts a strong constraint on the companion mass.

The blue and red Doppler shifted emission lines of $\mathrm{Ne}$ and $\mathrm{O}$ detected in the X-ray spectrum of $4 \mathrm{U}$ 1626-67 indicate that the lines originate in the accretion disk and there has been no significant variation in the widths of the emission lines (Krauss et al. 2007). The disk velocity component along our line-of-sight has a magnitude of a few hundred $\mathrm{km} \mathrm{s}^{-1}$. Therefore, the disk axis cannot be very close to our line-of-sight. One can assume that the orbital plane and the accretion disk plane are co-aligned. Otherwise, the forced precession of the accretion should result into a modulation of the $\mathrm{X}$-ray emission as a super-orbital period, which is not seen in the long term X-ray 


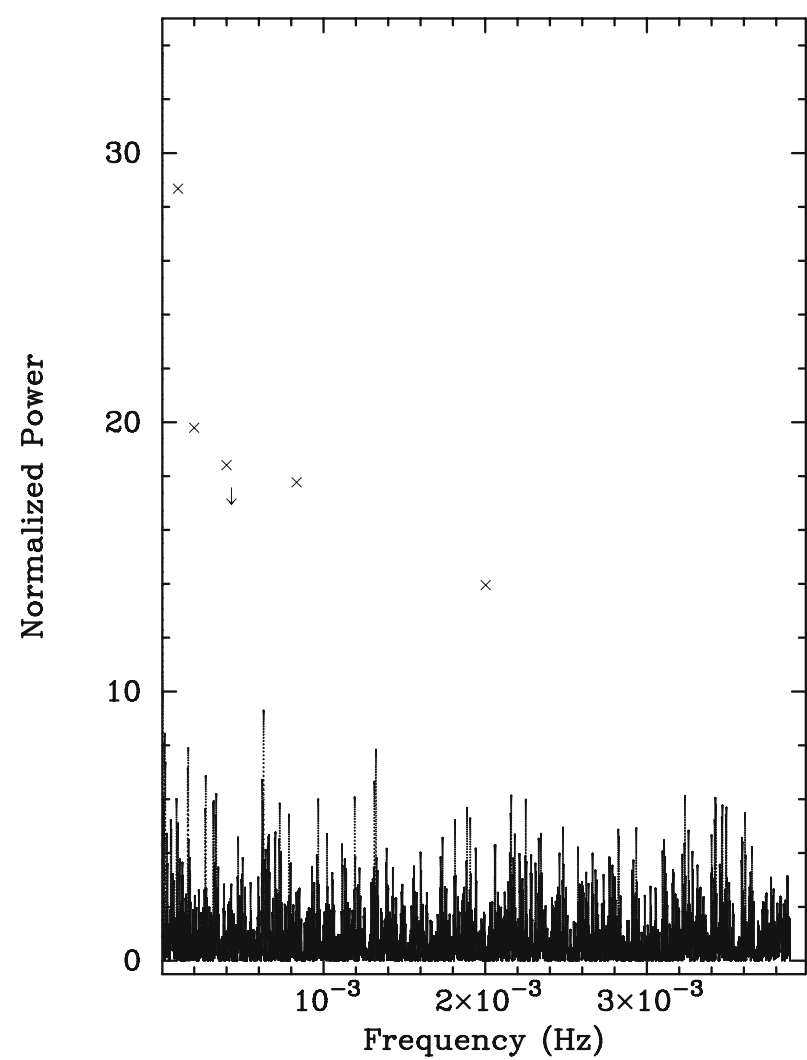

Figure 5. The normalized Lomb-Scargle periodogram for the pulse arrival time series of $4 \mathrm{U}$ 1626-67. Absence of a clear peak rules out the possibility of the presence of an orbital motion of the neutron star. With the addition of sinusoidal signals (of time period $500 \mathrm{~s}, 1200 \mathrm{~s}, 2500 \mathrm{~s}$, $5000 \mathrm{~s}$ and $10,000 \mathrm{~s}$ ) of amplitude $10 \mathrm{~ms}$, peaks were observed at the corresponding frequencies. The crosses in the figure indicate the peak power at those frequencies. The arrow marks the orbital period ( $2485 \mathrm{~s})$ as inferred from the optical observations.

light-curve of 4U 1626-67. The non-detection of orbital motion from pulse arrival time delay therefore leaves scope for only a very small mass companion star.

In view of the results presented here, we point out that $4 \mathrm{U} 1626-67$ is a strong candidate for a neutron star with a supernova fall-back accretion disk. Possibility of a supernova fall back accretion disk or fossil disk has been considered in many different contexts before. The possibility of supernova fall back accretion disk around neutron stars and black holes has also been found from modeling of SN explosion (Perna et al. 2000; Nomoto et al. 2006). Based on this model, Lin et al. (1991) explained the formation of planets around the radio pulsars. A unified picture for dim isolated thermal neutron stars, anomalous X-ray pulsars and soft gamma-ray repeaters can also be obtained from this model (Alpar et al. 2001). The spin down of anomalous $\mathrm{X}$-ray pulsars were also considered in the context of fall-back disk model (Chatterjee et al. 2000). All neutron stars can acquire debris through fall-back but the subsequent evolution of neutron star depends on the interaction between the stellar magnetic field and the disk flow (Chatterjee et al. 2000). Presence of a fall-back disk has been 


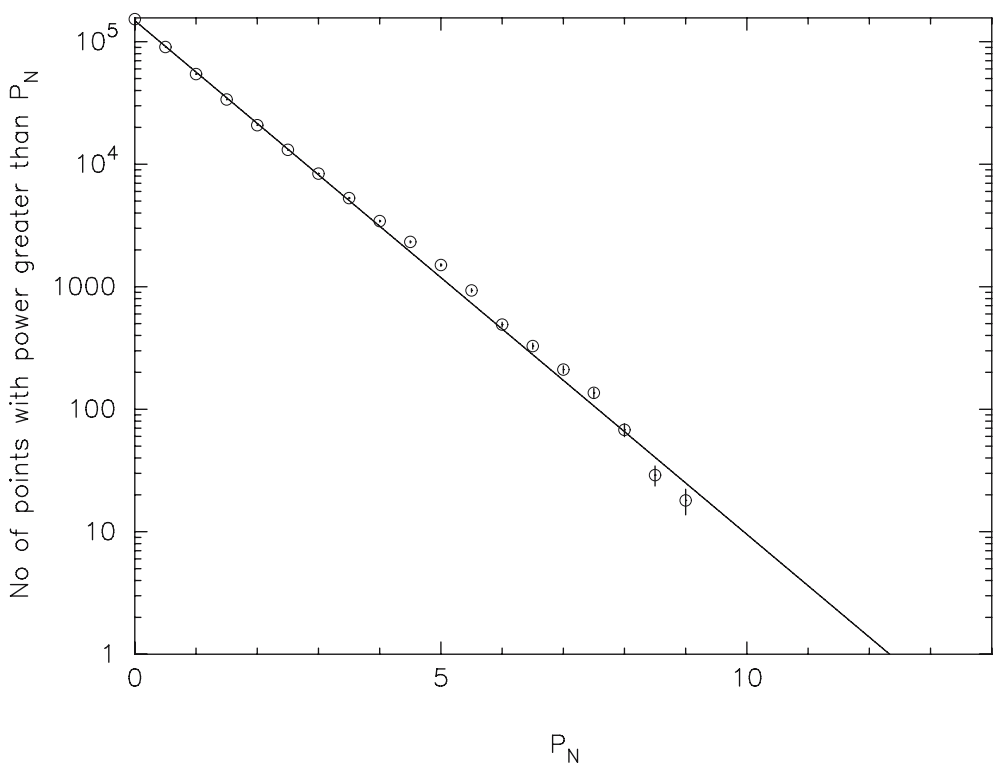

Figure 6. A plot of the normalized power distribution over all the frequencies in the Lomb-Scargle periodogram. The distribution is found to be exponential with highest power not exceeding 10.

proposed by Wang et al. (2006), around the anomalous X-ray pulsar 4U $0142+61$. However, Ertan et al. (2007) have suggested that the optical and near IR observations are consistent with the gaseous disk model. For a spin period of $7.66 \mathrm{~s}$ and magnetic field strength of $10^{12} \mathrm{G}$, a fossil accretion disk can feed the neutron star for a long period of time (Wang et al. 2006). If a significant fraction of the compact objects in core collapse supernovae have fall-back accretion disks, it influences the chemical evolution history of the Universe. However, since 4U 1626-67 seems to be only the second candidate for such a system, this does not give us any clue about what fraction of the neutron stars may have such a disk, how long the disks last, what fraction of the heavy metal falls back, etc. In this context, we also point out here that the accretion disk of the pulsar $4 \mathrm{U} 1626-67$ is indeed metal-rich.

\section{Acknowledgement}

We thank the RXTE team for making the data available and an anonymous referee for valuable comments on the manuscript.

\section{References}

Alpar, M. A. 2001, ApJ, 554, 1245.

Angelini, L., White, N. E., Nagase, F., Kallman, T. R., Yoshida, A., Takeshima, T., Becker, C., Paerels, F. 1995, ApJ, 449, L41.

Chakrabarty, D. et al. 1997, ApJ, 474, 414.

Chakrabarty, D., Homer, L., Charles, P. A., O’Donoghue, D. 2001, ApJ, 562, 985C.

Chatterjee, P., Hernquist, L., Narayan, R. 2000, ApJ, 534, 373. 
Ertan, U., Erkut, M. H., Eksi, K. Y., Alpar, M. A. 2007, ApJ, 657, 441.

Jahoda, K., Swank, J. H., Giles, A. B., Stark, M. J., Strohmayor, T., Zhang, W., Morgan, E. H. 1996, Proc. SPIE, 2808, 59.

Kaur, R., Paul, B., Kumar, B., Sagar, R. 2008, ApJ (in press) astro-ph/0801.1761.

Krauss, M. I., Schulz, N. S., Chakrabarty, D., Juett, A. M., Cottam, J. 2007, ApJ, 660, 605.

Levine, A., Ma, C. P., McClintock, J., Rappaport, S., Van Der Klis, M., Verbunt, F. 1988, ApJ, 327, 732 .

Lin, D. N. C., Woosley, S. E., Bodenheimer, P. H. 1991, Nature, 353, 827.

Middleditch, J., Mason, K., Nelson, J., White, N. 1981, ApJ, 244, 1001.

Nomoto, K., Tominaga, N., Umeda, H., Kobayashi, C., Maeda, K. 2006, Nucl. Phys. A., 777, 424.

Orlandini, M., Fiume, D., Frontera, F., del Sordo, S., Piraino, S., Santangelo, A., Segreto, A., Oosterbroek, T., Parmar, A. N. 1998, ApJ, 500, L163.

Paul, B., Agrawal, P. C., Mukerjee, K., Rao, A. R., Seetha, S., Kasturirangan, K. 2001, A\& A, 370, 529.

Rappaport, S., Markert, T., Li, F., Clark, G., Jernigan, J., McClintock, J. 1977, ApJ, $217,29$.

Perna, R., Hernquist, L., Narayan, R. 2000, ApJ, 541, 344P.

Schulz, N. S., Chakrabarty, D., Marshall, H. L., Canizares, C. R., Lee, J. C., Houck, J. 2001, ApJ, 563, 941S.

Shinoda, K., Kii, T., Mitsuda, K., Nagase, F., Tanaka, Y., Makishima, K., Shibazaki, N. 1990, PASJ, 42, L27.

Wang, Z., Chakrabarty, D., Kaplan, D. L. 2006, Nature, 440, 772. 